\title{
Variable structure strategy to avoid torque control saturation of a wind turbine in the presence of faults
}

\author{
C. Tutivén, Y. Vidal, L. Acho and J. Rodellar \\ Universitat Politècnica de Catalunya, \\ Applied Mathematics-III (MA-3) Department, CoDAlab, \\ Comte d'Urgell, 187, 08036, Barcelona, Spain. \\ Phone number:+0034934137309, e-mail: christian.tutiven@upc.edu, yolanda.vidal@upc.edu \\ leonardo.acho@upc.edu,jose.rodellar@upc.edu
}

\begin{abstract}
Every physical actuator is subject to saturation. It has been well recognized that, when the actuator saturates, the performance of the closed-loop system (designed without considering actuator saturation) may seriously deteriorate. In extreme cases, the system stability may even be lost. This paper proposes an avoid saturation strategy for the torque controller of a wind turbine benchmark model. The simulation results show that the proposed strategy has a clear added value with respect to the baseline controller (wellaccepted industrial controller) in the presence of faults. Another advantage of the contributed method is that conservative bounds for the actuator torque can be fixed in order to extend the service life of the wind turbine.
\end{abstract}

\section{Key words}

wind turbine, torque control, saturation, faults, fault detection

\section{Introduction}

Wind turbines (WT) have become an important source of renewable power generation in the last years, with a concurrent growth in demand for the expertise of engineers and researchers in the wind energy field. There are still many unsolved challenges in expanding wind power, and there are numerous open problems of interest to systems and control researchers. A major issue with wind turbines, specially those located offshore, is the relatively high cost of maintenance [1]. Since the replacement of main components of a WT is a difficult and costly affair, improved maintenance procedures can lead to essential cost reductions. Autonomous online fault detection algorithms allow early warnings of defects to prevent major component failures [2]. Furthermore, side effects on other components can be reduced significantly. Many faults can be detected while the defective component is still operational [2]. Thus necessary repair actions can be planned in time and need not be taken immediately and this fact is of special importance for off-shore plants where bad conditions can prevent any repair actions. Therefore the implementation of fault detection (FD) systems is crucial.

The past few years have seen a rapid growth in interest in wind turbine FD, [3]. So far, revising the literature, methods ranging from Kalman filters [4], observers [5], parity equations [6], dynamic weighting ensembles [7] and fuzzy modeling and identification methods [8] have already been suggested as possible model-based techniques for fault diagnosis of wind turbines.

For instance, in [9] and [10], besides of providing fault estimates, an active fault tolerant control approach is proposed. In [11] FD is based on the use of interval observers and unknown but bounded description of the noise.

In the presence of faults, if the torque controller requires excessive actuator action the closed-loop stability may be lost due to the torque saturation. The main possible effects of actuator saturation on a control system are poor performance and/or instability to large disturbances [12]. As a result, their stability cannot be guaranteed. Saturation mitigation techniques are using to avoid actuators saturation. For example, reference [13] presents the anti-windup technique. Direct approach to dealing with actuator saturation in wind turbines are used in [14] where an anti-windup controller to minimize de $H_{\infty}$ norm of the closed-loop system is designed. The design of a compensation method, which is based on variable structure systems to avoid both amplitude and rate input saturation by means of an auxiliary loop is presented in [15].

In this work, we propose a strategy to avoid torque saturation and the consequent undesired effects, guaranteeing the closed-loop operation of the control system. The proposed methodology is based on variable structure concepts and reference signal conditioning ideas. Our contribution: anticipatory activation, in contrast to immediate activation.

The structure of the paper is the following. In Section 2 the WT benchmark is recalled. In Section 3 the fault tolerant control used in the simulations is presented. Section 4 presents the design of the proposed avoid saturation strategy (ASS). The simulation results obtained with the proposed approach applied to the advanced WT benchmark are summarized in Section 5. Concluding remarks are given in Section 6.

\section{Reference wind turbine}

A complete description of the wind turbine model of the FDI/FTC benchmark can be found in [3]. Hereafter, only 
the generator-converter actuator model and the pitch actuator model will be shown, as the faults acting in the system affect those subsystems.

The generator and converter dynamics can be modeled by a first-order transfer function [3], which is given by:

$$
\frac{\tau_{g}(s)}{\tau_{g, r}(s)}=\frac{\alpha_{g c}}{s+\alpha_{g c}},
$$

where $\tau_{g}$ is the real generator torque, $\tau_{g, r}$ is the torque reference to the generator (given by the controller), where we set $\alpha_{g c}=50$ [16]. Additionally, the power produced by the generator, $P_{e}(t)$, can be modeled using [3]:

$$
P_{e}(t)=\eta_{g} \omega_{g}(t) \tau_{g}(t),
$$

where $\eta_{g}$ is the efficiency of the generator and $\omega_{g}$ is the generator speed. In the numerical experiments, $\eta_{g}=0.98$ is used [3]. The hydraulic pitch system consists of three identical pitch actuators, which are modeled as a linear differential equation with time-dependent variables, actual pitch angle $\beta_{i}(t)$ and its reference angle $\beta_{r_{i}}(t)$. In principle, it is a piston servo-system, which can be expressed as a second-order differential system [3]:

$$
\frac{\beta_{i}(s)}{\beta_{r_{i}}(s)}=\frac{\omega_{n}^{2}}{s^{2}+2 \zeta \omega_{n} s+\omega_{n}^{2}}, \quad i=1,2,3,
$$

where $\omega_{n}$ and $\xi$ are the natural frequency and the damping ratio, respectively. For the fault-free case, the parameters $\xi=$ 0.6 and $\omega_{n}=11.11 \mathrm{rad} / \mathrm{s}$ are utilized. [3].

\section{A. Baseline control strategy}

This paper deals with the full load region of operation. The baseline torque and pitch controllers specifications are described in the technical report [16] by the U.S. Department of Energy's National Renewable Energy Laboratory (NREL). Here a brief review of these controllers is given as its performance will be used for comparison with the proposed techniques.

In the full load region, the torque controller maintains constant the generator power; thus, the generator torque is inversely proportional to the filtered generator speed or,

$$
\tau_{g, r}(t)=\frac{P_{\mathrm{ref}}(t)}{\hat{\omega}_{g}(t)},
$$

where $P_{\text {ref }}$ is the reference power (normally the nominal value is used) and $\hat{\omega}_{g}$ is the filtered generator speed (see [16]). This controller will be referred to as the baseline torque controller. As the generator may not be able to supply the desired electromechanic torque depending on the operating conditions and in the case of overshooting, the torque controller is saturated to a maximum of 47.40 $\mathrm{KNm}$ and a maximum rate limit of $15 \mathrm{KNm} / \mathrm{s}$; see [16]. Therefore, if the torque controller required a fast and/or excessive actuator action the closed-loop operation could be lost because of these constraints, leading to large and long overshoots caused by windup and other undesired effects, or even the control system instability.
To assist the torque controller with regulating the WT electric power output, while avoiding significant loads and maintaining the rotor speed within acceptable limits, a collective pitch controller is added to the rotor speed tracking error. The collective blade pitch gain scheduling PI-controller (GSPI) is one of the first well-documented controllers, and it is used in the literature as a baseline controller to compare the obtained results [17]. This controller was originally developed by Jonkman for the standard land-based 5-MW turbine [16]. The GSPI control has the filtered generator speed, $\hat{\omega}_{g}(t)$, as the input and the pitch servo set-point, $\beta_{r_{i}}(t)$, as the output. That is,

$$
\begin{aligned}
& \beta_{r_{i}}(t)=K_{p}(\gamma)\left(\hat{\omega}_{g}(t)-\omega_{g, r}\right)+K_{i}(\gamma) \int_{0}^{t}\left(\hat{\omega}_{g}(\tau)-\omega_{g, r}\right) d \tau, \\
& K_{p}>0, K_{i}>0,
\end{aligned}
$$

where $\omega_{g, r}$ is the nominal generator speed (usually the nominal value is used) and the scheduling parameter $\gamma$ is taken to be the previously measured collective blade pitch angle. As the three pitch angles are measured, the collective pitch angle is obtained by averaging the measurements of all pitch angles. The pitch angle actuators generally present hard constraints on their amplitude and their speed response. Because of this, a pitch limit saturation to a maximum of $45^{\circ}$ and a pitch rate saturation of $8^{\circ} / \mathrm{s}$ are implemented (see [16]) to avoid pitch actuator damage.

\section{Fault-tolerant control}

The fault tolerant control (FTC) law based on a control plus disturbance estimator in the time discrete domain proposed in [18] is briefly recalled in this section. The control objective is the tracking of the reference signal $\beta_{r_{i}}(t)$ (given by the baseline pitch controller; see Equation (3) and its corresponding velocity, even in the case of pitch actuator fault. That is, the objective is to ensure the asymptotic convergence of the tracking error vector to zero. To achieve the sliding mode, the control law with disturbance compensator [19] is as follows:

$$
\begin{aligned}
& u[k]=-\hat{d}[k]+\left(c^{T} b\right)^{-1}\left[c^{T}\left(\begin{array}{c}
\beta_{r_{i}}[k] \\
\dot{\beta}_{r_{i}}[k]
\end{array}\right)-c^{T} A x[k]+q s[k]\right. \\
& -\eta \operatorname{sgn}(s[k])] \\
& \hat{d}[k]=\hat{d}[k-1]+\left(c^{T} b\right)^{-1} g[s[k]-q s[k-1] \\
& \quad+\eta \operatorname{sgn}(s[k-1])]
\end{aligned}
$$

where the notation $[k]$ is used for these discrete time signals, and $0 \leq q \leq 1,0<g<1$, and $\eta>0$ and being $\hat{d}[k]$ the fault estimator or also called the disturbance estimator. In the numerical simulations: $q=g=1 / 2$ and $\eta=100$. As can be seen in Equation (4), the proposed discrete controller for active FTC is dependent on a fault estimate, $\hat{d}[k]$, provided 
by the fault diagnosis system. The error vector is defined as:

$$
e[k]=\left(e_{1}[k], e_{2}[k]\right)^{T}=\left(\beta_{i}[k]-\beta_{r_{i}}[k], \dot{\beta}_{i}[k]-\dot{\beta}_{r_{i}}[k]\right)^{T},
$$

and

$$
s[k]=c^{T} e[k] .
$$

The pitch controller used by the FTC strategy is the baseline GSPI controller, see Section 2-A. On the other hand, the used torque controller is the chattering control proposed in [20], which is recalled here to be

$$
\begin{aligned}
\dot{\tau}_{c}(t)= & \frac{-1}{\hat{\omega}_{g}(t)}\left[\tau_{g, r}(t)\left(a \hat{\omega}_{g}(t)+\dot{\hat{\omega}}_{g}(t)\right)-a P_{\text {ref }}(t)\right. \\
& \left.+K_{\alpha} \operatorname{sgn}\left(P_{\mathrm{e}}(t)-P_{\text {ref }}(t)\right)\right]
\end{aligned}
$$

where $P_{\text {ref }}$ is the reference power and $P_{\mathrm{e}}$ is the electrical power considered here (only for the control design) to be described as [21]

$$
P_{\mathrm{e}}(t)=\tau_{g, r}(t) \hat{\omega}_{g}(t),
$$

where $\tau_{g, r}(\mathrm{t})$ is the torque control and $\hat{\omega}_{g}(t)$ is the filtered generator speed. In the numerical simulations the values $a=1$ and $K_{\alpha}=1.5 \cdot 10^{5}$ have been used and a first order approximation of $\dot{\hat{\omega}}_{g}(t)$ is computed. This torque controller is saturated to a maximum of $47.40 \mathrm{KNm}$ and a maximum generator torque rate saturation of $15 \mathrm{KNm} / \mathrm{s}$, similarly to the baseline one.

\section{Design of the Avoid Saturation Strategy (ASS)}

In the presence of faults, the torque controller might be saturated. This section describes the design of an avoid saturation strategy (ASS) based in a commutation law. Its main objective is to readjust the reference power $\left(P_{\text {ref }}\right)$ and the reference generator speed $\left(\omega_{g, r}\right)$ to avoid saturation and the consequent undesired effects, guaranteeing the closed-loop operation of the control system. Control torque bounds are added: the superior torque $\left(\tau_{s}\right)$ and the inferior torque $\left(\tau_{i}\right)$. These two values are chosen using the difference between the maximum mechanical torque $\left(\tau_{\max }\right)$ and the nominal torque $\left(\tau_{n}\right)$,

$$
\begin{aligned}
\tau_{\text {dif }} & =\tau_{\max }-\tau_{n}=47.40 \mathrm{KNm}-40.68 \mathrm{KNm} \\
& =6.72 \mathrm{KNm},
\end{aligned}
$$

and

$$
h=\frac{\tau_{\text {dif }}}{a} ; \quad a>0 .
$$

Different values of $a$ were tested and found that the ASS had better performance when $a=6$, so $h=1.12 \mathrm{KNm}$ and the proposed control bounds are:

$$
\tau_{s}=\tau_{n}+h=40.68 \mathrm{KNm}+1.12 \mathrm{KNm}=41.80 \mathrm{KNm},
$$

and

$$
\tau_{i}=\tau_{n}-h=40.68 \mathrm{KNm}-1.12 \mathrm{KNm}=39.55 \mathrm{KNm} .
$$

Fig. 1 illustrates the behavior of the ASS. When the control torque $\left(\tau_{g, r}\right)$ is greater than $\tau_{s}$, the ASS commutes $P_{\text {ref }}$ to the minimum power $\left(P_{\min }\right)$ and when $\tau_{g, r}$ is lower than $\tau_{i}$, the ASS commutes $P_{\text {ref }}$ to the maximum power $\left(P_{\max }\right)$, otherwise, $P_{\text {ref }}$ is equal to the nominal power $\left(P_{n}\right)$.

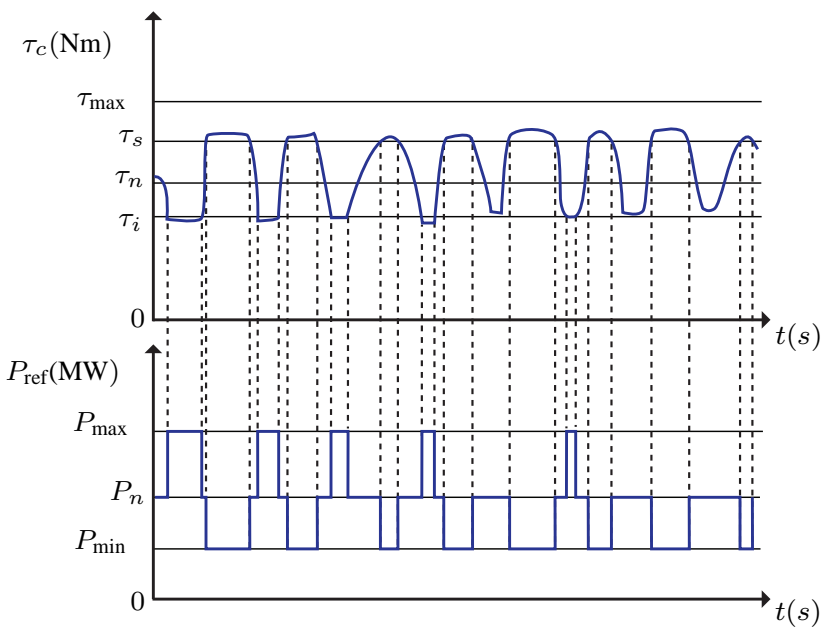

Fig. 1. Avoid saturation strategy.

The value of $P_{\max }$ and the value of the maximum generator speed $\left(\omega_{g, \max }\right)$ are established by using the simple rule of three to calculate $\omega_{g, \max }$,

$$
\frac{\tau_{\max }}{\tau_{n}}=\frac{\omega_{g, \max }}{\omega_{g, n}} ; \quad \omega_{g, \max }=143.22 \frac{\mathrm{rad}}{\mathrm{s}} .
$$

With $\tau_{\max }$ and $\omega_{g, \max }$, we calculate $P_{\max }$,

$$
P_{\text {max }}=\tau_{\max } \omega_{g, \max } ; \quad P_{\max }=6.78 \mathrm{MW} .
$$

To calculate the value of $P_{\min }$ and the value of the minimum generator speed $\left(\omega_{g, \min }\right)$, first we calculate the difference between $\tau_{n}$ and $\tau_{\text {dif }}$,

$$
\tau_{\min }=\tau_{n}-\tau_{\text {dif }}=33.95 \mathrm{KNm} .
$$

To calculate $\omega_{g, \min }$, we use again the rule of three,

$$
\frac{\tau_{\min }}{\tau_{n}}=\frac{\omega_{g, \min }}{\omega_{g, n}} ; \quad \omega_{g, \min }=102,59 \frac{\mathrm{rad}}{\mathrm{s}} .
$$

With $\tau_{\min }$ and $\omega_{g \text {,min }}$, we calculate $P_{\min }$,

$$
P_{\min }=\tau_{\min } \omega_{g, \min } ; \quad P_{\min }=3.48 \mathrm{MW} .
$$

So the ASS is responsible for performing the switching of $P_{r e f}$ and $\omega_{g, r}$, by the following implemented commutation law:

$$
P_{\mathrm{ref}}(t)=\left\{\begin{array}{ccc}
P_{\max }, & \text { if } & \tau_{g, r}(t)<\tau_{i}, \\
P_{\min }, & \text { if } & \tau_{g, r}(t)>\tau_{s}, \\
P_{n}, & \text { otherwise, }
\end{array}\right.
$$




$$
\omega_{g, r}(t)=\left\{\begin{array}{ccc}
\omega_{g, \max }, & \text { if } & \tau_{g, r}(t)<\tau_{i}, \\
\omega_{g, \min }, & \text { if } & \tau_{g, r}(t)>\tau_{s}, \\
\omega_{g, n}, & \text { otherwise. }
\end{array}\right.
$$

The block diagram of Fig. 2 shows the connections between the fault tolerant control (FTC) system presented in [18], with the added ASS.

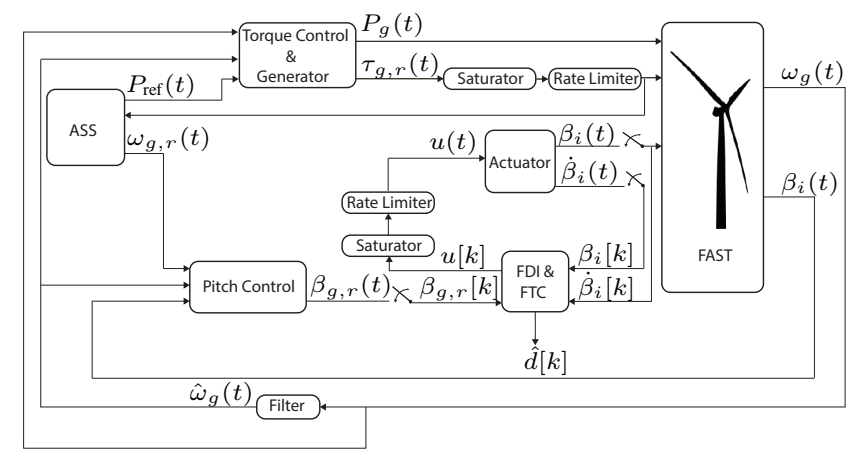

Fig. 2. Block diagram of the fault tolerant control with the avoid saturation strategy (FTC+ASS) closed loop system.

\section{Simulation results}

The results compare the performance of the contributed FTC+ASS technique under different faulty scenarios with respect to the baseline torque controller recalled in Section 2-A. The wind turbine simulator software FAST is used for simulations. The results compare the performance of the contributed FTC+ASS under different faulty scenarios with respect to the baseline control under the same faults.

The response of the generator velocity and electrical power are analyzed in terms of the normalized integral absolute error through the following performance indices:

$$
\begin{gathered}
J_{\omega}(t)=\int_{0}^{t}\left|\omega_{g}(\tau)-\omega_{g, n}\right| d \tau, \\
J_{p}(t)=\int_{0}^{t}\left|P_{g}(\tau)-P_{n}\right| d \tau .
\end{gathered}
$$

\section{A. Offset in generator speed sensor (F1)}

A faulty sensor can reduce turbine availability, especially if the sensor measurement is required for control. As discussed in [22], sensor failures can be difficult to diagnose.

Here, an offset fault in the generator speed sensor is studied. We selected an offset value of $15 \%$ of $\omega_{g, n}$ that is equal to $18,43 \frac{\mathrm{rad}}{\mathrm{s}}$. This fault, $(\mathrm{F} 1)$, is active all the time.

It can be seen from Fig. 3 that the system behavior (electrical power and generator speed) with the FTC+ASS is stable even when an offset fault exists in the generator speed sensor. On the other hand, the baseline system becomes unstable after a while when this type of fault exists.

As can be observed in Fig. 4 the proposed strategy avoids overloading $\tau_{g, r}$, protecting it from saturation and maintaining its values within the configured limits $\left(\tau_{s}\right.$ and $\left.\tau_{i}\right)$. Also the commutation of the nominal generator speed helps the
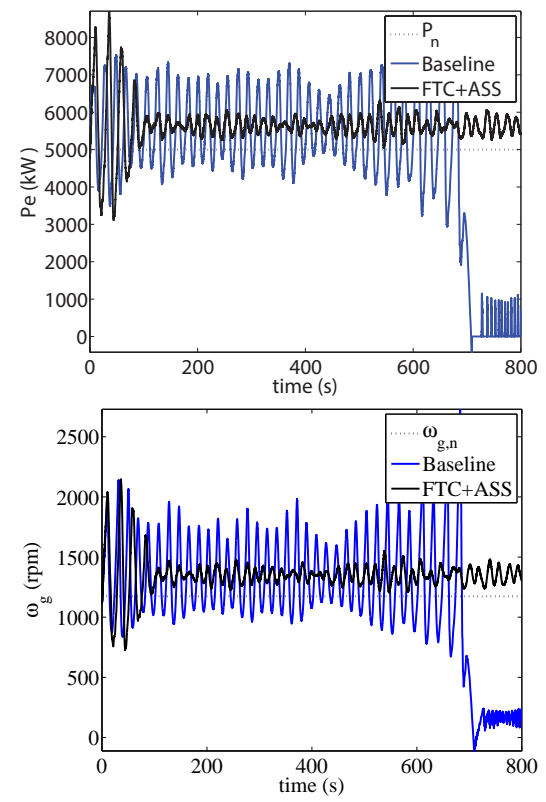

Fig. 3. Generated power (top) and generator speed (bottom) in case of offset fault (F1).

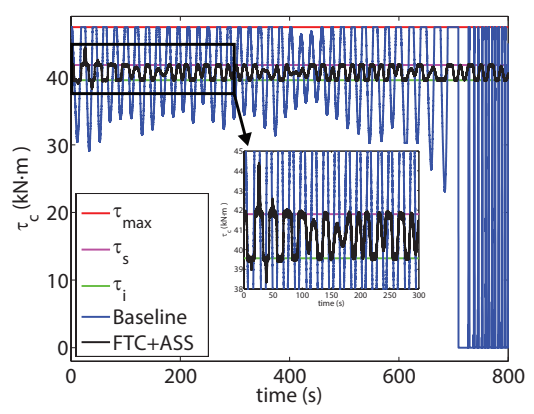

Fig. 4. Torque control in case of offset fault (F1).

blades pitch angles not to saturate as shown in the Fig. 5 . On the other hand, the baseline system becomes unstable, because of the saturation of torque and pitch controllers.

\section{B. Pitch actuator faults}

Blade pitch systems faults are studied as they have the highest failure rate in WT [17]. Some types of fault (high air content in oil (F2), pump wear (F3), and hydraulic leakage (F4)) may change the dynamics of the pitch system by varying the damping ratio $(\zeta)$ and natural frequencies $\left(\omega_{n}\right)$ from their nominal values to their faulty values in Equation (1). The parameters for the pitch system under different conditions are given in [3].

In the simulations, the faults are introduced only in the third pitch actuator (thus $\beta_{1}$ and $\beta_{2}$ are always fault-free) in the following way. From 0s to 100 s, it is fault free (FF). From 100s a 101s, a fault due to high air content in oil (F2) is linearly introduced. From 101s a 201s, F2 is fully active. From 201s a 202s, F2 is linearly eliminated. From 202s to 302 s, it is fault free. From 302 s a 322 s, a fault due to pump 

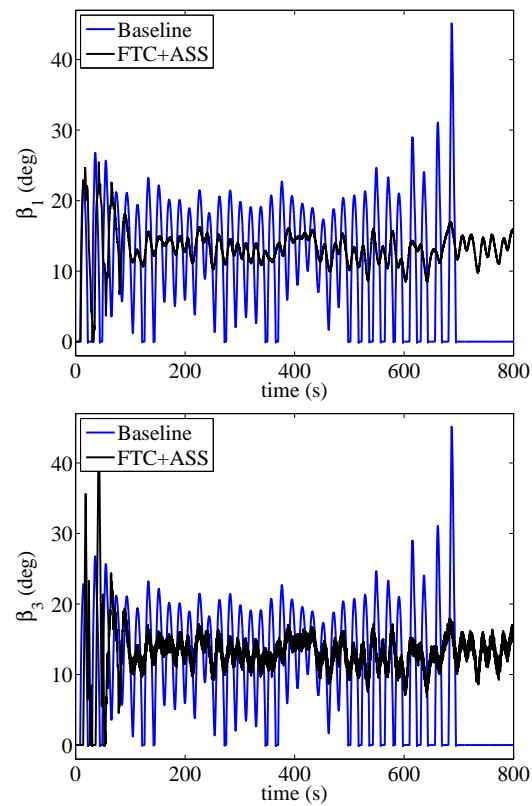

Fig. 5. Pitch angle variation of $\beta_{1}$ (top) and $\beta_{3}$ (bottom) in case of offset fault $(\mathrm{F} 1)$

wear (F3) is linearly introduced. From 322 s a 422 s, F3 is fully active. From 422 s a 442 s, F3 is linearly eliminated. From 442 s to 542 s, it is fault free. From 542 s a 562 s, a fault due to hydraulic leakage (F4) is linearly introduced. From 562 s a 662 s, F4 is fully active. From 662 s a 682 s, F4 is linearly eliminated. From 682 s to 800 s, it is fault free.

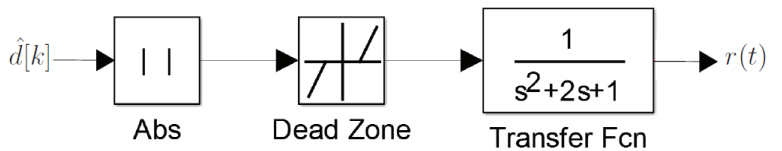

Fig. 6. Computation of the continuos residual signal $r(t)$. Note that the Simulink ${ }^{\circledR}$ dead zone block is used (start of dead zone value equal to 0 and end of dead zone value equal to 2000 .

The three types of faults are detected by the disturbance estimator $\hat{d}$ given in Equation 5. To finally setup the fault detection and isolation strategy, the proposed residual signal $r(t)$, is computed as described in Fig. 6 and its results are shown in Fig. 7. This residual signal is close to zero when the system is fault free. On the other hand, it is significantly affected when a fault appears, and it allows to isolate the type of fault (among the three studied pitch actuator faults).

It can be seen from Figures 8 (top) and 9 (top) that the FTC+ASS has a better performance of the electrical power and generator speed regulation compared to the baseline control. The performance indices $J_{p}$ and $J_{\omega}$ corroborate this statement, see Fig. 8 (bottom) and 9 (bottom).

As can be observed in Fig. 10, the proposed strategy has a better torque control performance than the baseline control, maintaining its values within the configured limits $\left(\tau_{s}\right.$ and $\left.\tau_{i}\right)$.

Fig. 11 (top) shows that the first pitch angle $\left(\beta_{1}\right)$, which

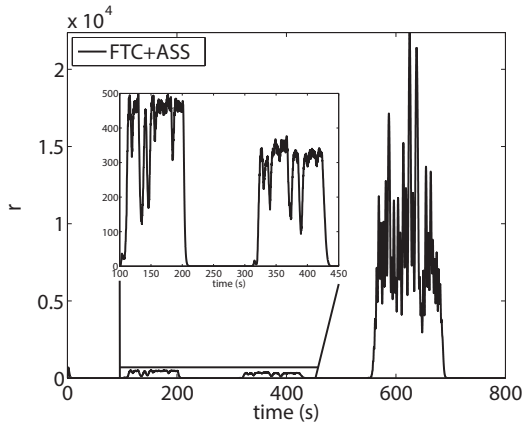

Fig. 7. Proposed residual signal in case of pitch actuator faults (F2, F3 and F4).
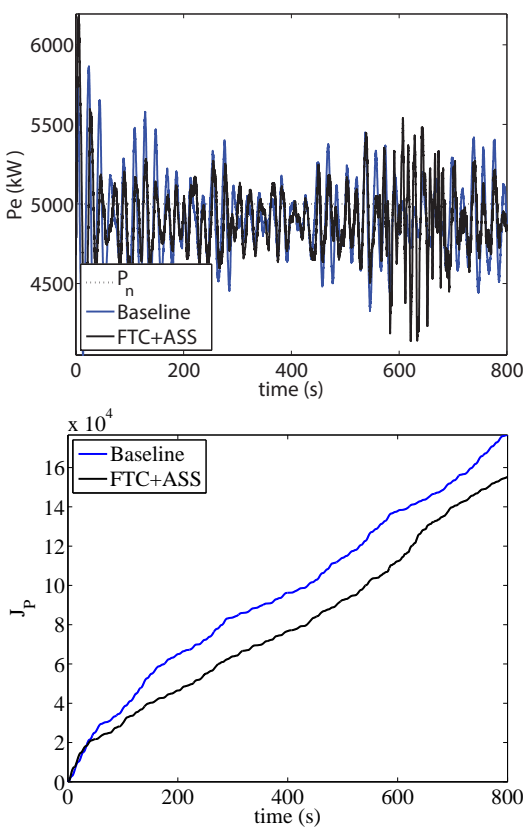

Fig. 8. Generated power (top) and $J_{p}$ index (bottom) in case of pitch actuator faults (F2, F3 and F4).

is always fault-free, has a slightly different behavior with the FTC+ASS than with the baseline control. This is due to the fact that the fault is introduced in the third pitch actuator $\left(\beta_{3}\right)$ as can be seen in the Fig. 11 (bottom). Although higher oscillations are present in the FTC+ASS, the pitch control signal is regulated within the authorized variation domain. That is, none of the variations exceed the mechanical limitations of the pitch actuator.

\section{Conclusions}

An avoid saturation strategy based on reference adaptation ideas and variable structure concepts is proposed to avoid the amplitude saturation of the torque controller in a $5 \mathrm{MW}$ wind turbine. The main objective is to reduce the actuator activity and avoid the instability of the system. On the other hand, the residual signal detects in short time the appearance of the faults in the pitch actuator. Moreover, according to the experimental results, the overall closed-loop system is robust against the studied faults. 

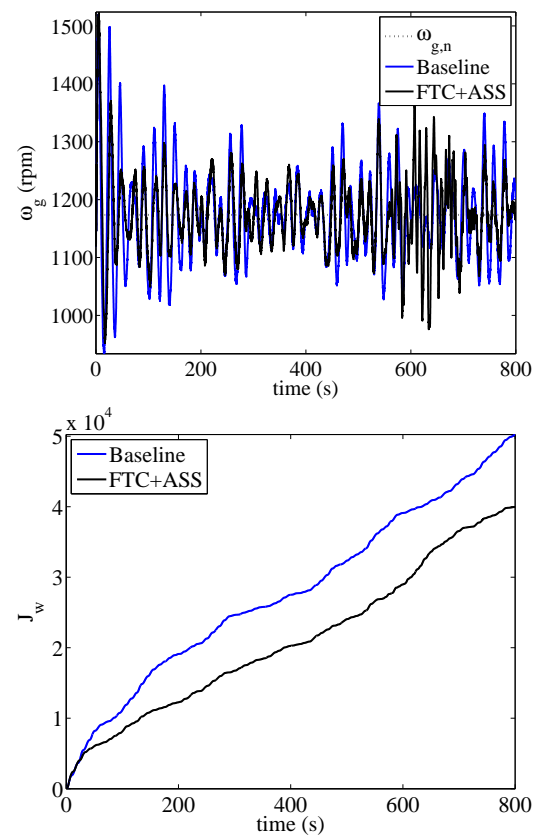

Fig. 9. Generator speed (top) and $J_{\omega}$ index (bottom) in case of pitch actuator faults (F2, F3 and F4).

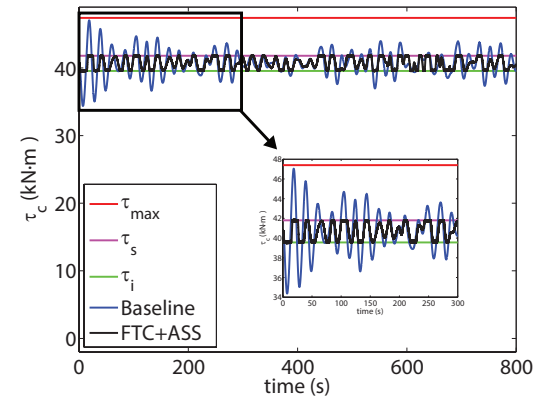

Fig. 10. Torque control in case of pitch actuator faults (F2, F3 and F4).

\section{Acknowledgments}

This work has been partially funded by the Spanish Ministry of Economy and Competitiveness through the research projects DPI2014-58427-C2-1-R, DPI2015-64170-R, and by the Catalonia Government through the research project 2014SGR859.

\section{References}

[1] C. P. Butterfield, W. Musial, J. Jonkman, P. Sclavounos, L. Wayman, Engineering challenges for floating offshore wind turbines, National Renewable Energy Laboratory Golden, CO, USA, 2007.

[2] Z. Hameed, Y. Hong, Y. Cho, S. Ahn, C. Song, Condition monitoring and fault detection of wind turbines and related algorithms: A review, Renewable and Sustainable Energy Reviews 13 (1) (2009) 1-39.

[3] P. F. Odgaard, K. E. Johnson, Wind turbine fault detection and fault tolerant control-an enhanced benchmark challenge, in: Proceedings of American Control Conference, IEEE, 2013, pp. 4447-4452.

[4] X. Wei, M. Verhaegen, T. Van den Engelen, Sensor fault diagnosis of wind turbines for fault tolerant, in: Proceedings of The International Federation of Automatic Control world congress, Vol. 17, 2008, pp. 3222-3227.

[5] P. F. Odgaard, J. Stoustrup, R. Nielsen, C. Damgaard, Observer based detection of sensor faults in wind turbines, in: Proceedings of European Wind Energy Conference, 2009, pp. 4421-4430.
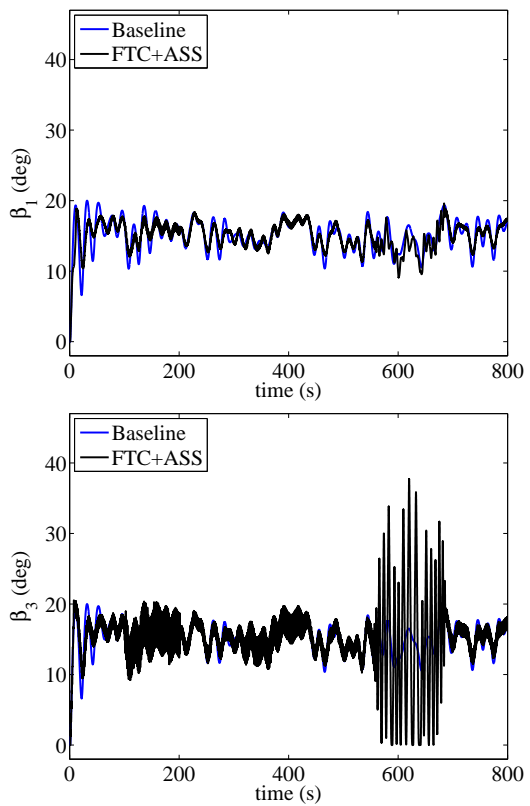

Fig. 11. $\beta_{1}$ (top) and $\beta_{3}$ (bottom) in case of pitch actuator faults (F2, F3 and F4).

[6] J. Blesa, V. Puig, J. Romera, J. Saludes, Fault diagnosis of wind turbines using a set-membership approach, in: Proceedings of The International Federation of Automatic Control world congress, 2011, pp. 8316-8321.

[7] R. Razavi-Far, M. Kinnaert, A multiple observers and dynamic weighting ensembles scheme for diagnosing new class faults in wind turbines, Control Engineering Practice 21 (9) (2013) 1165-1177.

[8] H. Badihi, J. S. Rad, Y. Zhang, H. Hong, Data-driven model-based fault diagnosis in a wind turbine with actuator faults, in: Proceeding of ASME 2014 International Mechanical Engineering Congress and Exposition, American Society of Mechanical Engineers, 2014.

[9] F. Shi, R. Patton, An active fault tolerant control approach to an offshore wind turbine model, Renewable Energy 75 (2015) 788-798.

[10] M. S. Shaker, R. J. Patton, Active sensor fault tolerant output feedback tracking control for wind turbine systems via T-S model, Engineering Applications of Artificial Intelligence 34 (2014) 1-12.

[11] J. Blesa, D. Rotondo, V. Puig, F. Nejjari, FDI and FTC of wind turbines using the interval observer approach and virtual actuators/sensors, Control Engineering Practice 24 (0) (2014) 138 - 155.

[12] W. S. Levine, Control system fundamentals, CRC press, 1999.

[13] L. Zaccarian, A. R. Teel, Modern anti-windup synthesis: control augmentation for actuator saturation, Princeton University Press, 2011.

[14] M. Meisami-Azad, K. M. Grigoriadis, Anti-windup linear parametervarying control of pitch actuators in wind turbines, Wind Energy 18 (2) (2015) $187-200$.

[15] F. Garelli, P. Camocardi, R. J. Mantz, Variable structure strategy to avoid amplitude and rate saturation in pitch control of a wind turbine, International Journal of Hydrogen Energy 35 (11) (2010) 5869-5875.

[16] J. Jonkman, S. Butterfield, W. Musial, G. Scott, Definition of a 5 MW reference wind turbine for offshore system development, Tech. rep., National Renewable Laboratory (NREL), Golden, Colorado (2009).

[17] R. Chaaban, D. Ginsberg, C. Fritzen, Structural load analysis of floating wind turbines under blade pitch system faults, in: N. Luo, Y. Vidal, L. Acho (Eds.), Wind Turbine Control and Monitoring, Advances in Industrial Control, Springer International Publishing, 2014 , pp. $301-334$.

[18] Y. Vidal, C. Tutivén, J. Rodellar, L. Acho, Fault diagnosis and faulttolerant control of wind turbines via a discrete time controller with a disturbance compensator, Energies 8 (5) (2015) 4300-4316.

[19] Y. Eun, J.-H. Kim, K. Kim, D.-I. Cho, Discrete-time variable structure controller with a decoupled disturbance compensator and its application to a CNC servomechanism, IEEE Transactions on Control Systems Technology 7 (4) (1999) 414-423. 
[20] Y. Vidal, L. Acho, N. Luo, M. Zapateiro, F. Pozo, Power control design for variable-speed wind turbines, Energies 5 (8) (2012) 3033-3050.

[21] B. Boukhezzar, L. Lupu, H. Siguerdidjane, M. Hand, Multivariable control strategy for variable speed, variable pitch wind turbines, Renewable Energy 32 (8) (2007) 1273 - 1287.

[22] K. E. Johnson, L. J. Fingersh, A. D. Wright, Controls advanced research turbine: lessons learned during advanced controls testing, National Renewable Energy Laboratory, 2005. 